\title{
IL DISCORSO INDIRETTO LIBERO NEL ROMANZO DI GIORGIO BASSANI IL GLARDINO DEI FINZI-CONTINI: FUNZIONI TESTUALI E CARATTERISTICHE LINGUISTICHE
}

\section{Considerazioni introduttive}

Quando la sezione letteraria della commissione statale per l'italiano aveva scelto il romanzo Il giardino dei Finzi Contini (GFC) come testo su cui preparare le conoscenze letterarie per l'esame di maturità in italiano lingua straniera nei licei sloveni non si aspettava particolari difficoltà di comprensione da parte degli studenti. La fruizione però si è rivelata meno soddisfacente del previsto. L'attenta analisi linguistica delle sue caratteristiche strutturali svolta in seguito allo scopo di offrire poi agli insegnanti suggerimenti concreti per la presentazione dei punti problematici in classe ha portato alla scoperta di una insospettata complessità, soprattutto di natura narrativa e sintattica. Questo compito apparentemente pratico ha spronato una ricerca di respiro più ampio, che ha implicato analisi sistematiche della prima e dell'ultima versione del romanzo, ${ }^{1}$ nonché di una serie di traduzioni in varie lingue. ${ }^{2}$

Nel caso del GFC si tratta di un'opera stilisticamente assai elaborata, con alta concentrazione di tecniche narrative e di stratagemmi retorici. Così si osserva una grande frequenza di ritorni all'indietro da un dato punto temporale alle fasi anteriori (flash back), di preparazioni del terreno per l'azione centrale tramite un uso particolare di forme verbali (preludio), di anticipazioni di azioni appartenenti a un periodo posteriore (flash forward), e soprattutto di discorso indiretto libero (da ora in poi DIL). Proprio su quest'ultimo - un espediente retorico che in sloveno, per cause strutturali, viene realizzato in modo diverso, è meno adoperato da parte degli autori ed è del tutto trascurato nell'insegnamento scolastico ${ }^{3}$ - si concentra la presente discussione. ${ }^{4}$ Va sottolineato inoltre che la trattazione del DIL non è inclusa nel programma di studio nemmeno nel caso di insegnamento dell'italiano come lingua seconda al livello avanzato.

1 Per le modificazioni apportate dalla I alla II e infine alla III versione, si veda Kanduth 1983.

2 Sono state prese in considerazione la traduzione slovena, tedesca, inglese, francese, americana e spagnola.

3 Le interviste fatte alle matricole universitarie confermano regolarmente la loro non familiarità con il fenomeno, mentre la maggioranza di loro non conosce nemmeno il termine.

4 Nel riportare il materiale illustrativo mi riferisco all'ultima edizione/ristampa del 1996. In caso di uso della prima versione il numero della pagina nelle parentesi rotonde che segue il brano illustrativo sarà preceduto da "ANT". 


\section{Discorso indiretto libero}

Il discorso indiretto libero (DIL) costituisce uno dei modi espositivi fondamentali, insieme alla diegesi (cioè il racconto della voce narrante, l'ultimo narratore) e ad altri tipi di discorsi, ${ }^{5}$ principalmente quello diretto (DD) e quello indiretto (DI). II DIL è stato studiato da molti punti di vista e in varie tradizioni linguistiche, mi baso tuttavia sull'inquadramento teorico proposto da Mortara Garavelli $(1985,1995)$. Questo tipo di discorso riportato sembra presentare i fatti contemporaneamente da due punti di vista diversi: da quello della voce narrata, del personaggio - cioè dell'enunciatore originario (L1) - e da quello della voce narrante (L). La realizzazione formale di questo mezzo stilistico è affidata al vario combinarsi di indici di diversi piani (lessico, espressività, deissi personale, deissi temporale e locale, forme verbali, frase o elemento citante ecc.) orientati su uno dei due centri discorsivi (cioè di L e di L1).

In quanto "indicatore di polifonia", non sorprende che questo mezzo paradossale prenda tanta parte nel romanzo in $1^{\mathrm{a}}$ persona: Bassani ha affidato il racconto all'io narrante, che è in grado di far rivivere i discorsi delle persone narrate (l'io narrato incluso) solo attraverso la propria memoria, determinata inevitabilmente da un punto di vista diverso da quello del produttore di ogni singolo discorso ricordato. L'impiego iconico per le situazioni appartenenti al passato delle forme verbali considerate "del passato" (imperfetto (IM), trapassato prossimo (TP), condizionale passato (CC) ecc.) - tipiche della diegesi del passato - ha la funzione di distanziare dall'attualità della voce narrante i discorsi delle voci narrate, vivaci e immediati come sono nella loro conformazione sintattica e lessicale (si pensi alle interiezioni, esclamazioni, domande e ordini riportati ecc.) e nell'uso della deissi temporale e locale ("oggi", "qui"). Bassani si serve del DIL per presentare il contenuto di atti mentali di ogni tipo - come del resto testimoniano espliciti elementi citanti oppure come suggerisce il contesto: si tratta ad es. di discorsi "effettivamente" proferiti dall'io narrato o da altri personaggi (cominciai a raccontare (135); esclamò, con enfasi ironica (75)); scritti (scrissi (125); Si trattava di una letterina spiritosa [...] Micòl esordiva con lo scusarsi [...] aggiungeva (124)); discorsi sognati (soggiungevo [...] conveniva (106)); riflessioni (mi dicevo (61)); pensieri ascritti ad altri (parevano dirsi tutti e quattro (29); mi diceva con gli occhi (51)); previsioni (mi dicevo (154)); ricordi (Avevo previsto tutto con molta esattezza (154)); percezioni (116), fantasticherie (pensavo (47)) e persino visioni (già lo vedevo (151)).

\subsection{Caratteristiche formali del DIL canonico}

Se persino i test condotti tra gli studenti universitari sloveni hanno mostrato che le occorrenze dei discorsi indiretti liberi nel romanzo lasciano spesso sconcertati anche quelli che vantano una buona padronanza della lingua italiana, ciò si spiega in parte con il fatto che in sloveno, per ragioni strutturali, il DIL differisce pochissi-

5 Uso generalmente questo termine ombrello per riferirmi anche al pensiero, ricordo, fantasticheria e simili. 
mo dal discorso diretto - cambia, se necessario, solo la deissi personale: ${ }^{6}$ come mezzo stilistico risulta poco efficace ed è quindi poco usato. Nemmeno l'insegnamento dell'italiano al livello avanzato prevede la presentazione del suddetto procedimento, mentre la ricca varietà di realizzazione dei DIL concreti nel romanzo di Bassani non permette, senza una guida opportuna, di scoprirne le linee di orientamento. ${ }^{7}$

Ora, in italiano, nella realizzazione canonica del DIL rispetto al DD e al DI del "corrispondente" DD si mantengono, oltre che le caratteristiche lessicali e sintattiche, anche la deissi temporale e locale, mentre del DI si ha una redistribuzione dei ruoli della deissi personale e un probabile cambiamento nel tempo verbale. Ad es.

DD Non te lo posso dire, rispose. Perché, lo saprò solo domani.

DIL Non glielo poteva dire, rispose. Perché l'avrebbe saputo solo domani.

DI Rispose che non glielo poteva dire perché l'avrebbe saputo solo il giorno seguente/l'indomani).

Ma non necessariamente la $1^{\mathrm{a}}$ e la $2^{\mathrm{a}}$ persona confluiscono nella $3^{\mathrm{a}}$, come sopra. ${ }^{8}$ Nel DIL, come nel DI, la deissi personale aseconda i cambiamenti nella distribuzione dei ruoli nella costellazione comunicativa: parlante (IO), ascoltatore (TU), nessuno dei due (ad es. LEI). Illustro quanto sopra con i seguenti DI tratti dal romanzo:

Senonché la notizia che ebbi da mia madre mentre uscivo dallo sgabuzzino del telefono, e cioè che verso mezzogiorno Micòl Finzi-Contini aveva telefonato chiedendo di me ("Mi ha pregato di dirti che è dovuta partire per Venezia, che ti saluta, e che ti scriverà", aggiunse la mamma, guardando altrove), fu sufficiente per farmi di colpo cambiare avviso. (114)

6 Il principio sloveno mantiene il tempo verbale inalterato sia nel DI che nel DIL: così, ad esempio, a un DD con un "futuro sloveno" ("Ne BOM te ZAPUSTILA", mu je rekla. /"Non ti LASCERD", gli disse./) corrispondono un DIL (Ne BO ga ZAPUSTILA, je rekla. /Non lo LASCERA, gli disse./) e un DI (Rekla mu je, da ga ne BO ZAPUSTILA. /Gli disse che non lo LASCERA./) con altrettanti "futuri sloveni".

7 Va messo in rilievo il fatto che nel libro di testo usato - Contesti italiani di M. Pichiassi e G. Zaganelli -, sebbene numerosi brani testuali (circa un quinto dei testi riportati) presentino occorrenze di DIL, non c'è nessun riferimento a questa procedura retorica. Con il termine discorso indiretto libero viene invece commentato quello che è in reaità pensiero diretto. Il commento a p. 125: "Discorso indiretto libero. La storia è raccontata da un narratore esterno, in terza persona, ma in alcune parti il punto di vista da esterno (quello del narratore) si fa interno, e la vicenda allora è vista con gli occhi della ragazza. Il punto di vista interno trova la sua realizzazione sul piano sintattico e stilistico nella forma del discorso indiretto libero. In questo caso, ad esempio, le riflessioni e i pensieri della ragazza sono riportati liberamente senza alcuna dipendenza sintattica, come continuazione della narrazione." si riferisce alla situazione testuale a p. 121: "Non osò pensare: l'ha lasciata qui perché io la vedessi.", dove sia "lei" di "non osò" che "io vedessi" si riferiscono a un'unica persona, quella del titolo "La ragazza del sabato sera".

8 Secondo J. Toporišič, autore della più autorevole grammatica slovena, tra le caratteristiche dell'espediente in questione ci sarebbe appunto l'uso della $3^{\mathrm{a}}$ persona; cfr. Toporišič 2000: "[...] povedano podamo v 3. osebi, spremni stavek pa večinoma opustimo: to je polpremi govor." (p. 653); "V polpremem govoru se podaja vsebina pogovora nekako neposredno iz spremljanja toka zavesti udeleženca pogovora; vse je podano v 3. osebi;" (659); " Pri polpremem govoru je potencialni primarni govor izražen tako, da je govoreči in ogovorjeni skozi zavest motrečega (ki se sploh ne izdaja kot govoreči) odtujen v 3. osebo." (707/708). 
Mentre il riferimento alla persona narrata (e narrante), chiamiamola "G.", nel discorso di Micòl alla madre di G. era stato necessariamente realizzato con la $3^{\mathrm{a}}$ persona ("lo saluto"), nel discorso della madre questo riferimento è fatto con la $2^{\mathrm{a}}$ persona ("ti saluta"), e nella diegesi, infine, con la $1^{a}$ persona ("chiedendo di me"). La trasformazione parte qui dalla $3^{\mathrm{a}}$ persona e attraverso la $2^{\mathrm{a}}$ approda alla $1^{\mathrm{a}}$.

Similmente nel discorso di Adriana, seguito dalla diegesi di G.:

"Sarà il caso di spiegargli" disse. [...] cominciò quindi a raccontarmi [...]

Si veda anche 2.1 .2 sotto.

È vero che nel romanzo sono ampiamente rappresentati casi canonici con la frase citante presente nel co-testo, le forme verbali e la deissi personale orientata sulla voce narrante e la deissi temporale, locale e dimostrativi orientati sul produttore del discorso originario, ad es.:

In fondo Perotti ERA un brav'uomo, pensavo. ERA contento anche lui che la signorina FOSSE TORNATA a casa. Gli SI POTEVA dar torto, povero vecchio? D'ORA in poi AVREBBE certamente SMESSO di brontolare. (157)

Ma la varietà di combinazioni di vari parametri, tanto nei DIL quanto nella diegesi è veramente notevole e non permette a un non specialista di scoprirne le regolarità o almeno di intuirne le linee di tendenza. Passerò quindi in rassegna alcuni parametri più importanti.

\subsubsection{Inclusione del DIL nel co-testo}

Nell'ultima edizione, quella a disposizione del lettore odierno, il DIL è spesso difficilmente riconoscibile, separato com'è dalla frase citante semplicemente da una virgola, un punto e virgola o un punto, ad es.:

Alzò una mano.

"Sarà il caso di spiegargli" disse.

Sbuffò, levò gli occhi al cielo.

Era capitato un fatto molto antipatico; cominciò quindi a raccontarmi in tono da maestra, mentre uno dei ragazzi più giovani tornava a schiacciare il piccolo aguzzo pulsante di corno nero del campanello d'ingresso. Va bene, io non lo sapevo, ma lei e Bruno nel torneo sociale di chiusura, [...] (62)

Nell'edizione originaria infatti vi stavano i trattini: il DIL era messo graficamente in risalto, facilitando di molto la comprensione: ${ }^{9}$

9 I trattini sono mantenuti solo quando l'elemento citante interrompe la frase, come si vede nel penultimo e nel terzultimo esempio illustrativo nel 2.1.1. 
Ne fu visibilmente dispiaciuta; ma la prospettiva di poter comunicarmi qualcosa di importante, qualcosa su cui evidentemente mancavo di notizie, travolse subito in lei ogni altro pensiero.

Era capitata una cosa molto "antipatica" - cominciò a raccontare, mentre uno dei due ragazzi più giovani tornava a schiacciare il piccolo, aguzzo pulsante di corno nero del campanello d'ingresso -. Forse non lo sapevo, ma lei e Bruno, nel torneo sociale che si era testè concluso al Circolo del Tennis, [...] (ANT 82)

Come constatato per la situazione generale (cfr. Mortara Garavelli), anche nel $G F C$ i DIL vengono introdotti nel co-testo in svariati modi: accompagnati dal verbo presentativo (o da un'analoga espressione rivelatrice) che li precede o segue o viene loro intercalata. Altre volte il DIL si trova in una completiva - subordinata sintatticamente ma non semanticamente-, per cui a volte sembrerebbe di trovarci davanti a un DI, quando invece si tratta di un DIL, come suggeriscono appunto gli elementi costitutivi orientati su L1:

Dopodiché, cambiando di nuovo argomento, mi chiese se per caso, POCO FA, non fossi passato in bicicletta lungo la Mura degli Angeli. (111)

Aggiunse che da molto tempo in QUA il mio modo di condurmi non era dignitoso: né per me, né per lei. (194)

Ammise poi che anche lui, da un po' di tempo in QUA, si era accorto che Alberto non stava bene. (216)

Data la generale oscillazione nell'uso dei deittici (v. sotto) anche negli indubbi DIL del $G F C$, in certi casi è impossibile decidere dove termini un DI e dove cominci un DIL. Ma forse la decisione non è nemmeno necessaria: l'effetto di indeterminatezza che ne consegue sembra riflettere la difficoltà di distinguere tra l'intensità delle diverse voci, ad es:

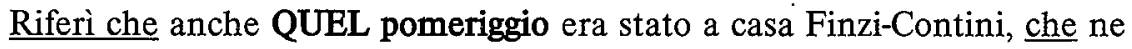
veniva proprio ADESSO (204)

Il pensiero che l'INDOMANI pomeriggio, lui, il fortunato, avrebbe certamente veduto Alberto e Micòl, parlando forse di me con loro, bastava a farmi dimettere ogni velleità di ribellione, a costringermi dentro il mio guscio. (210)

Dissi che QUELLA sera non potevo, ma che l'INDOMANI sarei passato quasi di certo da casa sua, alla solita ora. Se però vedeva che tardavo aggiunsi - non mi aspettasse. In tal caso ci saremmo incontrati da Giovanni. Non era da Giovanni che lui sarebbe andato a cena? (232)

Il romanzo contiene anche un numero di DIL con altri discorsi al loro interno, DD, DI e persino DIL. Ad es. sono DIL "al secondo grado", accompagnati sempre da un co-testo presentante, per menzionarne soltanto alcuni, il discorso immagina- 
rio degli etruschi nei pensieri dell'io narrato (dovevano dirsi, p. 7; quello dell'inserviente della Biblioteca Comunale nel discorso dell'io narrato (aveva ripetuto, p.135) o il discorso del professore tedesco nel racconto di Micòl (Era stato quanto mai esplicito, il "degno messere", p. 156).

In alcuni casi il co-testo successivo reinterpreta come DIL la parte del testo letta in un primo momento come narrazione diegetica. Così nell'esempio seguente, nel capoverso inserito tra due repliche in DD, il DIL esplicitato da "Come adesso - riflettevo" presuppone una prima parte del pensiero e la fa individuare appunto in quanto precede: si tratta quindi di un DIL (contenente al suo interno un altro DD).

"Sicuro" continuò lui, alzando un poco la voce. "Che cosa avresti voluto fare? Fidanzarti?"

Anche Micòl, quella sera in camera sua, mi aveva rivolto la stessa domanda. Aveva detto: "Cos'è che avresti preteso? Che ci fidanzassimo, scusa?". Io non avevo fiatato. Non avevo avuto niente da rispondere.

Come adesso - riflettevo - come adesso con mio padre.

"Perché no?" feci tuttavia, e lo guardai. (228)

Altre volte la presenza del DIL viene suggerita dal lessico e dal senso espresso. Così ad es. nella versione originale la parte del testo inserita in un capoverso tra due repliche in $\mathrm{DD}$ sembrerebbe a prima vista il commento diegetico dell'io narrante, ma le espressioni e il senso di inferiorità dell'enunciatore che ne traspare, sono la spia del pensiero del ragazzo di una volta: ${ }^{10}$

"Esageri, però", disse. "Che cosa vuoi che conti avere una materia a ottobre?"

Ma mi prendeva in giro, evidentemente, e un poco anche mi disprezzava. Era abbastanza normale, in fondo, che un fatto simile fosse capitato a un tipo come me, figlio di gente così comune, talmente "assimilata": a un quasi-goi, insomma. Che diritto avevo di far tante storie?

"Credo che tu ti faccia delle idee un po' strane", risposi. (52)

\subsubsection{Deissi personale}

La deissi personale nei DIL del GFC - riflessa nelle proforme personali (io, mio) e nelle marche nelle forme verbali ${ }^{11}$ - segue coerentemente l'orientamento sulla voce narrante $(\mathrm{L})$ : le persone presentate sono viste dalla sua prospettiva. Illustro con le imperative, la prima diretta all'io narrato (passaggio dalla $2^{\mathrm{a}}$ persona alla $1^{\mathrm{a}}$ ), la seconda al suo amico (passaggio dalla $2^{\mathrm{a}}$ persona alla $3^{\mathrm{a}}$ ):

GUARDASSI invece il giovane Lattes, per favore. (54)

10 Il traduttore sloveno, ad es., non aveva riconosciuto gli indici semantici e ha offerto ai lettori, in luogo del pensiero dell'io narrato - ragazzo, il commento diegetico dell'adulto.

11 Sono escluse le forme verbali con funzione di segnale discorsivo (cfr. 2.1.4.1.1). 
Però STESSE molto attento, lui, Malnate, [...] STESSE molto attento, lui, a non lasciarsi fregare dalla falsa apparenza di bonarietà di quel suo faccione plebeo. $(136 / 7)$

Vanno menzionate qui due deviazioni: evidentemente errori tipografici. ${ }^{12}$ Così è sbagliata la prima persona singolare in Gli avevo detto, magari (64) riferito, nel DIL del personaggio Bruno Lattes, al segretario del G.U.F., quindi "aveva detto"; errata è anche la prima persona plurale, nel DIL di Micòl, in Eccome se ci eravamo arrivati (87), quando parla di sé e del fratello, quindi "erano arrivati". Lo stesso vale inoltre per la svista in senso opposto: la terza persona nell'autoriferimento dell'io narrante nel discorso indiretto senza darmi il tempo di avvertirlo che aveva scambiato frasi con entrambi (202), invece di "avevo scambiato". Nella prima versione, la deissi personale nei casi citati è usata correttamente. Vista la complessità del procedimento analizzato, tali errori gravano non poco sulla comprensione.

\subsubsection{Deissi temporale e locale}

Accanto a numerosi casi di DIL costruiti con la deissi temporale, locale o i dimostrativi orientati sul centro discorsivo del personaggio (ad es. oggi, qui, poco fa, questa), ad es.

[...] la responsabilità della loro ATTUALE impotenza BISOGNAVA accollarla proprio a quei bravi, degni decorativi galantuomini in cilindro e stiffelius $[\ldots](128)$

nel GFC si trovano anche numerose occorrenze di DIL con l'orientamento su L:

Ma come lo vedeva, lui che parlava tanto di tesori di rettitudine, bontà eccetera, un caso successo a me, proprio a me, appena poche mattine AVANTI? (135)

A questo proposito va osservato che alcuni casi di DIL con deissi locale e temporale che nella versione originale erano orientate verso $\mathrm{L}$, nell'ultima sono stati corretti e presentano l'orientamento su L1. Ecco tre casi di autocorrezione:

Era stato Alberto a dirgli che OGGI sarei venuto a trovarlo. [...] Senonché Micòl in casa OGGI (versione originale (ANT 149): QUEL GIORNO) non c'era [...] essendo purtroppo dovuta partire IERI pomeriggio per Venezia. $(117 / 8)$

Eh, sì, pensavo: STASERA (versione originale (ANT 60): QUELLA SERA), rincasando, il papà mi avrebbe magari picchiato. Però io le sue botte potevo ormai affrontarle tranquillamente. (46)

12 Nella ristampa del 1996 della terza versione, permangono una ventina di errori tipografici che, configurandosi come parole di senso compiuto, non sono sempre di facile riconoscimento, ad es. giorno invece di giro, anzianità invece di arianità ecc. 
A casa, $O R M A I$ - pensavo - avevano di certo già saputo: da Otello Forti, magari. (35) (versione originale: $A$ QUELL'ora, certo - pensavo - a casa mia avevano già saputo: da Otello Forti, probabilmente. (ANT 49))

In altre situazioni analoghe, la prima scelta rimane tuttora immutata:

E non aggiunsi altro, ripreso di colpo dall'angoscia dell'inevitabile incontro con mio padre. QUELLA sera stessa, al più tardi, sarei dovuto tornare a casa. Non avevo altra scelta. (43)

Lei ne approfittò per alzare il ricevitore e dire in cucina che le portassero pure la cena: ma TRA una mezzoretta, non prima, giacché - tornò a ripetere - QUELLA sera non aveva "niente fame". (181)

L'ultimo esempio illustrativo contiene già all'interno di uno stesso DIL l'oscillazione tra i due orientamenti ( $\mathrm{L} 1 \mathrm{e} \mathrm{L}$ ), che del resto si riscontra in tutto il romanzo. Si confronti la distribuzione della coppia di dimostrativi questo/quello (v. inoltre sotto i casi con ora/allora, qui/li, scorso/precedente, ecc.):

[...] e QUEST'anno [...] chissà se a Ca' Foscari ci avrebbe mai messo piede (50)

$\mathrm{E}$ non vedevo come fosse invecchiato, in QUELL'ultimo anno? (51)

\subsubsection{Espressioni deittiche nella DIEGESI}

A rendere ancora più complesso il quadro dell'uso dei deittici nel romanzo sono, nella diegesi, gli usi anaforici delle espressioni come ora/adesso e occorrenze di deissi testuale. Infatti, accanto a enunciati diegetici, orientati verso il quadro deittico della voce narrante, con allora:

[...] e soltanto ALLORA mi accorsi che lui mi guardava perplesso, pentito.

(me ne rendevo perfettamente conto anche ALLORA) (206)

l'autore usa la coppia allora/adesso con funzione anaforica per creare diversi strati di profondità temporale e quindi di pertinenza:

Avevamo sempre parlato di molte cose, ALLORA, [...].

ADESSO invece, per telefono, i nostri discorsi tornavano di continuo su loro $[. .].(103)$

La diegesi è piena di adesso e ora anaforici:

ADESSO eravamo molto riguardosi l'uno con l'altro, perfino troppo. (214)

Così nel testo globale essi appaiono nella diegesi con la funzione deittica orientata sulla voce narrante:

[...] così intima, così riparata, starei per dire così sepolta, soprattutto così adatta al me stesso d'allora, ADESSO lo capisco!, a proteggere quella specie di pigra brace che è tante volte il cuore dei giovani. (140) 
con la funzione anaforica:

ADESSO lo scrosciare della pioggia sopra il tetto della rimessa aveva cessato di essere udibile. (93)

E ADESSO pensavo - senza che nemmeno a QUESTO pensiero il mio cuore accelerasse i suoi battiti [...] (237)

nei DIL:

Ma ADESSO, invece? mi chiedevo sconsolato. Che cosa m'importava di andare a casa loro, ADESSO, se Micòl non ce l'avrei più trovata? (114) Ma certo. Come lo capivo, ORA, quel suo gesto nel bordello di via delle Volte (238)

e, nell'ambito del DIL, nella presentazione della visione al procedimento storico:

Strano e terribile [...] scorgere da lontano, nascosto nell'ombra, mio padre che toma proprio ADESSO dal Circolo dei Negozianti [...] (47/8)

Similmente con la coppia qui/qua e li/là. Nel DIL i due tipi si alternano nella funzione deittica:

Adesso pensavo che sì, se dopo tutto era QUA, da Micòl, che Giampi Malnate veniva ogni notte dopo avermi lasciato sulla soglia. (238)

E poi, sul serio, ancora più che a me aveva pensato ad Alberto, il quale tranne con Giampiero Malnate, era rimasto QUA senza nessuno con cui scambiare ogni tanto due chiacchiere. (179)

Indicai le schiere dei lattimi [...]: gli unici oggetti, LÌ dentro - dissi - che nel sogno mi fossero apparsi diversi da quel che erano nella realtà. (175)

e nella diegesi con funzione anaforica:

Dimenticavo però di dire che di quadri, QUI, a differenza che in tutte le altre stanze della casa, [...] non se ne vedeva che uno. (146)

LÌ, forse, c'era una quantità maggiore di quadri dell'Ottocento [...] (118)

(QUI alzò una mano, come a prevenire una mia eventuale smentita) (226)

Di Lì a poco uscì dal bagno, sbarbato e sorridente. (204)

Qui appare nel $G F C$ anche con la funzione di deissi testuale:

La mia storia con Micòl Finzi-Contini termina QUI. (240)

\subsubsection{Altre oscillazioni}

L'autore oscilla anche nella scelta tra i verbi venire e andare: nel DIL del professor Ermanno e del padre del protagonista privilegia il primo, nel DIL di Alberto, invece, il secondo:

Era stato Alberto a dirgli che OGGI sarei VENUTO a trovarlo. (117) 
Era già stato abbastanza curioso quello che era successo la settimana SCORSA al Tempio, per Roshashanà (io non avevo voluto VENIRci, al solito: e una volta di più avevo fatto male). (55)

E tutti i pomeriggi erano buoni, se la faccenda mi interessava, aveva aggiunto. OGGI, DOMANI, DOPODOMANI: potevo ANDARE quando volevo, portando con me chi mi pareva, e anche il sabato, SI CAPISCE. $(29 / 30)$

Nell'esempio seguente si ha un accostamento di ora con funzione anaforica nella diegesi e l'oscillazione, nel DIL, tra l'orientamento su L (di dieci anni PRIMA) e l'orientamento su L1 (questa):

ORA, però, mi sentivo opprimere da un disagio, da un'amarezza, da un dolore quasi insopportabili. Della bambina di dieci anni PRIMA - mi chiedevo disperato - che cosa era rimasto in QUESTA Micòl di ventidue anni [...]? (107)

L'oscillazione si osserva anche nell'impiego dei termini della coppia scorso/precedente, che si alternano nel DIL:

Era già stato abbastanza curioso quello che era successo la settimana SCORSA al Tempio, per Roshashanà [...] (55)

Va bene, io non lo sapevo, ma lei e Bruno, nel torneo sociale di chiusura, cominciato appunto a metà della settimana PRECEDENTE, avevano raggiunto nientemeno che la finale [...] (62)

Ma il deittico scorso appare anche in funzione anaforica. Se nel primo caso si potrebbe scorgere una specie di DIL, e pertanto l'orientamento su L1, molto più difficile è invece giustificare il secondo impiego nell'indubbia diegesi:

Gli raccontai quindi con minuzia e senza tacere dell'episodio del maggio SCORSO, in camera sua, episodio che io ritenevo, dissi, determinante in senso negativo, e irrimediabile. (222)

Sembrava quasi che l'inverno non volesse più andarsene. $\mathrm{E}$ anch'io, il cuore abitato da un oscuro, misterioso lago di paura, mi aggrappavo alla scrivanietta che il professor Ermanno dal gennaio SCORSO aveva fatto collocare per me sotto la finestra di mezzo del salone del biliardo [...] (142)

Sono ugualmente insoliti i due impieghi del deittico tuttora nella diegesi. Bisogna però sottolineare che nel secondo caso si tratta di una modificazione stilistica, forse non sufficientemente attenta, di un neutrale ancora della prima versione:

[...] la mia restava una speranza vaga [...] di ricongiungermi a lei e ai luoghi paradisiaci dai quali TUTTORA mi si escludeva. (198)

Ormai mi dirigevo verso lo sbocco di corso Ercole I d'Este, ormai, pedalando lungo il muro di cinta, ero giunto in vista del portone, e Alberto 
faceva TUTTORA risuonare il suo "olifante". "Bada, non svignartela!" dicevano adesso i suoi fischi sempre potentissimi [...] (187)

\subsubsection{Forme verbali}

È tipico per le forme verbali personali del DIL il loro orientamento sulla voce narrante. Se la diegesi è nel procedimento fondamentale - cioè se vengono usate le forme del set del passato, raggruppate intorno al passato remoto - anche nel DIL si usa questo tipo di forme e i protagonisti si riferiranno alle azioni nel loro passato, attualità e avvenire, invece che per es. con passato prossimo, presente e futuro, con trapassato prossimo, imperfetto e condizionale composto, per limitarci alle situazioni più tipiche e tralasciando per il momento modificazioni modali, temporali e aspettuali. Tale è appunto la situazione nel GFC. Si vedano in proposito gli esempi illustrativi in altre sezioni. Interessanti in questo contesto sono gli enunciati non dichiarativi, cioè interrogativi, imperativi e esclamativi, che, modificati nella forma verbale, mantengono l'intonazione della variante diretta:

Non ERA da Giovanni che lui SAREBBE ANDATO cena? (233)

Per quanto riguarda la forma verbale nelle imperative, in sostituzione dell'imperativo si ha l'imperfetto del congiuntivo:

(LASCIASSI stare Leopardi, per carità! Leopardi ERA un'altra cosa, e poi AVEVA SCRITTO la Ginestra, NON me ne DIMENTICASSI...) (209)

\subsubsection{Forme verbali del "SET DI BASE"}

Nei DIL del GFC appaiono inoltre anche le forme verbali dell'altro set, quello "di base" (presente $(\mathrm{Pr})$, passato prossimo (PP), futuro $(\mathrm{F})$, condizionale $(\mathrm{C})$, imperativo ecc.). I motivi sono per lo più i seguenti: le forme verbali hanno la funzione di segnali discorsivi, si riferiscono a fatti extratemporali o ancora validi per la voce narrante o sono usati nel "procedimento storico".

\subsection{Forme verbali con funzione di SEGNALE DISCORSIVO}

Nei DIL del romanzo incontriamo le seguenti forme con funzione di segnale discorsivo:

- imperativo vero e proprio, $2^{\mathrm{a}}$ pers. sg.: va' là (106), guarda (104),

- forme esortative, $1^{a}$ pers. pl. cong. pres.: siamo giusti (53), vediamo (188), parliamoci chiaro (172) intendiamoci $(125,213)$, diciamolo pure $(104,105,129)$, figuriamoci $(63,70,132,184,225)$, lasciamo perdere (137), andiamo (196), ad es.:

Che cosa gli mancava per progredire? Vediamo. Gambe? (188)

- forme di $1^{a}$ pers.sg. e pl. del pres. ind.: se vogliamo (166); (b) non dico (137): 
Ogni qualvolta tornavo dopo una settimana di assenza - proseguì - dichiarando, non so, che ero stato a Roma, [...] m'illudevo forse che Alberto e Malnate non lo capissero [...] (196)

- forme di $2^{\mathrm{a}}$ pers. sg. pres.: hai voglia (238)

- forme di $3^{\mathrm{a}}$ pers. sg. pres.: non è che $(184)$, è vero $(68,84,104,159)$, è chiaro $(70)$, va bene $(55,62,136,143)$, sta bene (216), si capisce (15), si vede (104), si sa (128).

Le stesse espressioni possono essere usate con il loro significato comune e allora nel DIL appaiono all'imperfetto: era vero $(130,180)$; era chiaro, era evidente (203). Si confrontino le due funzioni del sintagma verbale nel DIL:

Era vero, o no - mi aveva chiesto subito, trascurando qualsiasi preambolo [...] - Era vero, o no, che io e "tutti gli altri" [...] eravamo stati dimessi in blocco dal club: "cacciati via", insomma? (49)

OGGI, è vero - aveva ammesso - noi ci saremmo limitati a "sopraluogare" soltanto là in fondo, dalla parte del tramonto [...] (84)

\subsection{Situazioni extratemporali}

Come nei DI in genere anche nei DIL, le azioni considerate dalla voce narrante di validità generale vengono presentate con le forme del set di base:

Macché. Appena POTEVO, io, al contrario, le VENIVO addosso con baci e altro, come se non lo SAPESSI che in situazioni come la nostra non c'è niente di più antipatico e controindicato. (195)

Altrettanto si può riscontrare per le verità cristalizzate nei proverbi, motti e modi di dire, come ad es. Tutto il male non viene per nuocere. (181) e Non si sa mai. (117). La formula del proverbio con l'uso del passato prossimo si rileva inoltre nella seguente citazione: ${ }^{13}$

Neppure i quadri di Morandi lo PERSUADEVANO, diceva: cose fini senza dubbio delicate, ma secondo lui troppo "soggettive" e "disancorate". La paura della realtà, la paura di sbagliare: ecco ciò che ESPRIMEVANO in fondo le nature morte di Morandi, i suoi famosi quadri di bottiglie e di fiorellini; e la paura, anche in arte, è sempre STATA una pessima consigliera... Al che, non senza esecrarlo in segreto, io non trovavo mai argomento da opporre. $(209 / 210)$

\subsubsection{3 "PROCEDIMENTO STORICO"}

Le forme del set di base appaiono nel DIL del GFC anche quando viene presentata la percezione diretta di azioni teliche. Ne è un esempio il momento più intenso della fantasticheria dell'io ragazzo, quando cioè l'immaginazione prende la forma di

13 Cfr. L'uso del PP nel proverbio I diciott'anni non SON mai STATI brutti (Selene 1990, p. 122). 
una vera e propria visione. Tutta la parte concernente il padre viene riportata al procedimento storico (così viene risolto il problema del "presente riportivo" nel DIL); dopodiché l'autore riprende il procedimento fondamentale (potevo). Il brano esibisce inoltre altri due casi dell'uso del set di base (con funzione di segnale discorsivo (si capisce) e per designare situazioni atemporali (sono, non è rimasto)):

Ma non ERA mica detto che non POTESSI uscire all'aperto mai più! Durante il giorno DORMIVO, SI CAPISCE, interrompendo il sonno quando SENTIVO sfiorarmi le labbra dalle labbra di Micòl, e più tardi riaddormentandomi con lei fra le braccia. Di notte, tuttavia, di notte POTEVO benissimo fare delle lunghe sortite, specie se SCEGLIEVO le ore dopo l'una, dopo le due, quando tutti SONO a dormire, e per le vie della città non E RIMASTO in pratica nessuno. Strano e terribile, ma dopo tutto anche divertente, passare da via Scandiana, rivedere la nostra casa, la finestra della mia camera da letto adattata ormai a salotto, scorgere da lontano, nascosto nell'ombra, mio padre che TORNA proprio ADESSO dal Circolo dei Negozianti, e non gli PASSA nemmeno per la testa che io SONO vivo e STO OSSERVANDOlo. Diffatti TIRA fuori di tasca la chiave, $A P R E, E N T R A$, e quindi tranquillo, proprio come se io, suo figlio maggiore non SLA mai ESISTITO, RINCHIUDE il portone d'un colpo solo.

E la mamma? Non POTEVO tentare un giorno o l'altro di far sapere almeno a lei, per tramite di Micòl, magari, che non ERO morto? (47/8)

L'espediente retorico è ripetuto più tardi, quando nell'ambito della presentazione (al DIL) dei pensieri dell'io narrato durante la cena di pasqua questi ha una visione. $\mathrm{Al}$ momento cruciale l'autore passa dal procedimento fondamentale a quello storico:

Io non ero morto - mi dicevo - io ero ancora ben vivo! Ma allora, se ancora vivevo, perché mai restavo lì insieme con gli altri, a che scopo? [...] $\mathrm{Ne}$ avrebbe avute, da raccontare! Fino a mezzanotte, fino all'una, fino alle due! E poi? Poi ci sarebbe stata la scena ultima, quella degli addii. Già la vedevo. ERAVAMO SCESI tutti in gruppo giù per le scale buie, come un gregge oppresso. Giunti nel portico, qualcuno (forse io) ERA ANDATO avanti, a socchiudere il portone di strada, ed ora, per l'ultima volta, prima di separarci, SI RINNOVAVANO da parte di tutti, me compreso, i buonanotte, gli auguri, le strette di mano, gli abbracci, i baci sulle gote. Senonché improvvisamente, dal portone rimasto mezzo aperto, là, contro il nero della notte, ecco irrompere dentro il portico una raffica di vento. $\grave{E}$ vento d'uragano, e VIENE dalla notte. PIOMBA nel portico, lo ATTRAVERSA, OLTREPASSA fischiando i cancelli che SEPARANO il portico dal giardino, e intanto $H A$ DISPERSO a forza chi ancora VOLEVA trattenersi, HA ZITTITO di botto, col suo urlo selvaggio, chi ancora INDUGLAVA a parlare. Voci esili, gridi sottili, subito sopraffatti. Soffiati via, tutti: come foglie leggere, come pezzi di carta, come capelli di una chioma incanutita dagli 
anni e dal terrore... Oh, Ernesto in fondo ERA STATO fortunato a non poter fare l'università in Italia. (151/152)

\section{CONCLUSIONE}

L'alta concentrazione dei vari mezzi retorici nel romanzo Il giardino dei FinziContini si era rivelata un serio ostacolo alla fruizione dell'opera da parte di studenti sloveni, sebbene provvisti di una preparazione linguistica di livello avanzato.

Le analisi sistematiche del romanzo, in particolare del massiccio impiego del discorso indiretto libero (DIL), hanno confermato che questo espediente retorico non si presenta come un fenomeno formalmente unitario e di automatica riconoscibilità, distinguibile sempre dai DI e dalla narrazione diegetica, bensì come un mezzo stilistico realizzato in forma di diverse combinazioni di indici su vari piani linguistici: contenuto, espressività, lessico, deissi personale, deissi temporale e locale, paradigmi verbali, sintassi ecc.

Agli studenti che nella loro madrelingua non conoscono analoghi principi espressivi, le constatazioni relative, oltre che alla funzione testuale dei DIL nel $G F C$, anche alla loro conformazione e alle loro modalità di inserimento nel co-testo dovrebbero agevolare la decodifica del messaggio globale del romanzo, aiutandoli al contempo a raggiungere una più completa conoscenza del funzionamento della lingua italiana.

\section{BIBLIOGRAFIA}

BÄUERLE, R. (1979), Temporale Deixis, temporale Frage. Tübingen, Niemeyer.

BASSANI, G. (1991), Il giardino dei Finzi-Contini. Milano, Arnoldo Monđadori.

BAZZANELLA, C. (1995), "I segnali discorsivi". In: Grande grammatica italiana di consultazione, III, L. Renzi, G. Salvi, A. Cardinaletti (edd.). Bologna, Il Mulino, 225-257.

BERTINETTO, P. M. (1991), "Il verbo." In: Grande grammatica di consultazione, II, L. Renzi, G. Salvi (edd.). Bologna, Il Mulino, 113-161.

BRINTON, L. (1980), “'Represented perception': a study in narrative style”. Poetics 9, 363-381.

BRON!ZWAER, W. J. M. (1970), Tense in the Novel. (An Investigation of Some Potentialities of Linguistic Criticism.) Groningen, Wolters-Noordhoff.

CARTAGO, G. (1993), "Un uso particolare dell'indiretto libero". Studi di Grammatica Italiana 15, 157-167.

CONTE, M.-A. (1992), "Deixis textuelle et Deixis am Phantasma". In: Texte, Sätze, Wörter und Moneme. Festschrift für Klaus Heger zum 65. Geburtstag, S. R. Anschütz (ed.). Heidelberg, Heidelberger Orientverlag, 153-161

Conte, M.-A. (1996), "Dimostrativi nel testo: tra continuità e discontinuità referenziale." Lingua e stile 31, 135145.

D'ANGELO, M. (1994), "Alcuni aspetti semantici del discorso riportato e l'analisi degli spazi mentali". Lingua e stile 29, 3-24.

FLEISCHMAN, S. (1990), Tense and Narrativity. London, Routledge.

FLUDERNIK, M. (1996), "Linguistics and literature: Prospects and horizons in the study of prose". Journal of Pragmatics 26, 583-611.

GENETTE, G. (1994), Die Erzählung. München, Wilhelm Fink. 
GREWENDORF, G. (1982), "Deixis und Anaphorik im deutschen Tempus". Papiere zur Linguistik 26, 47-83.

HEINERMANN, Theodor (1931), Die Arten der reproduzierter Rede. Münster, Achendorffsche Verlagsbuchhandlung.

HERCZEG, G. (1963), Lo stile indiretto libero in italiano. Firenze, Sansoni.

HINRICHS, E. (1986), "Temporal Anaphora in Discourses of English". Linguistics and Philosophy 9, 63-82.

KANDUTH, E. (1983), "Giorgio Bassanis Il giardino dei Finzi-Contini im Spiegel der Varianten". Italienische Studien 6, 105-123.

KLEIN, W. (1977), "Die Wissenschaft der Interpretation". In: Methoden der Textanalyse, W. Klein (ed.). Heidelberg, Quelle \& Meyer, 1-23.

LEECH, G. N./ M. H. SHORT (1981), Style in fiction. London/ New York, Longman.

LO CASCIO, V. (1986), "Temporal Deixis and Anaphor in Sentence and Text: Finding a Reference Time". In: Temporal Structure in Sentence and Discourse, V. Lo Cascio, C. Vet (edd.). Dordrecht, Foris, 191-227.

MIKLIČ,$T$. (1997), "Segnalazione della temporalità nel testo: che cosa aiuta il ricevente a collocare le azioni sull'asse temporale". In: Atti del Terzo Convegno della Società Internazionale di Linguistica e Filologia Italiana, L. Agostiniani et al. (edd.). Napoli, Edizioni Scientifiche Italiane, 477-505.

Miklič, T. (1998), Uso cataforico del trapassato prossimo italiano: un espediente testuale per la messa in rilievo. Linguistica XXXVIII, 183-195.

Miklič, T. (1999), Kontrastiranje realizacija jednog retoričkog postupka: slobodni neupravni govor (DIL) u datom italijanskom tekstu i u njegovim prevodima na slovenački, nemački i engleski jezik. In: Kontrastivna jezička istraživanja: VI simpozijum, Novi Sad, maj 1998: zbornik radova. Novi Sad, Filozofski fakultet, 277-288.

Miklič, T. (2002), Su alcuni usi tempo-aspettuali dei paradigmi verbali italiani TRAPASSATO PROSSIMO e IMPERFETTO. Linguistica XLII, 47-64.

Miklič, T. (i.c.s. a), Interpretazione della funzione testuale dei paradigmi verbali italiani: tentativo di un modello d'analisi integrato. (XXXV Convegno SLI, Parigi 2001).

Miklič, T. (i.c.s. b), Alcune caratteristiche testuali del romanzo Il giardino dei Finzi Contini di Giorgio Bassani: confronto di due versioni. (III Convegno degli Italianisti Europei, Cracovia 2001).

Miklič, T. (i.c.s. c), Testo letterario in classe d'italiano L2. Come aiutare lo studente ad esplorare il lato espressivo dell'opera per capirla e gustarla meglio. (XII Convegno nazionale GISCEL, Cagliari 2002: "Educazione linguistica e educazione letteraria: intersezioni e interazioni").

MORTARA GARAVELLI, B. (1985), La parola d'altri. Palermo, Sellerio editore.

Mortara Garavelli, B. (1995), "Il discorso riportato". In: Grande grammatica italiana di consultazione, III, L. Renzi, G. Salvi, A. Cardinaletti (edd.). Bologna, Il Mulino, 427-468.

OLTEAN, S. (1995), "Free indirect discourse: some referential aspects". Journal of Literary Semantics 24, 21-41.

PALACAS, A. L. (1993), "Attribution Semantics: Linguistic Worlds and Point of View". Discourse Processes 16, 239-277.

PARTEE, B. (1984), "Nominal and Temporal Anaphora". Linguistics and Philosophy 7, 243-286.

PICHIASSI, M./G. ZAGANELLI ( $\left.{ }^{3} 2003\right)$, Contesti italiani. Viaggio nell'italiano contemporaneo attraverso $i$ testi. Materiali per la didattica dell'italiano L2. Perugia, Guerra Edizioni.

REYES, G. (1993), Los procedimientos de cita: estilo directo y estilo indirecto. Madrid, Arco Libros.

SCHIFFRIN, D. (1981), "Tense variation in narrative". Language 57, 45-62.

SELENE, A. (1990). Dizionario dei proverbi. Milano, Orsa Maggiore.

SMITH, C. (1981), "Semantic and Syntactic constraints on Temporal interpretation". Syntax and Semantics 14, 213-237.

TOPORIŠIČ, J. (2000), Slovenska slovnica. Četrta, prenovljena in razširjena izdaja. Maribor, Založba Obzorja.

VANELLI, L. (1995), "La deissi". In: Grande grammatica italiana di consultazione, III, L. Renzi, G. Salvi, A. Cardinaletti (edd.). Bologna, Il Mulino, 261-375.

WEINRICH, H. (1964), Tempus: Besprochene und erzählte Welt. Stuttgart, Kohlhammer. 
Polpremi govor se v nekaterih slovenskih besedilih sicer pojavlja, vendar ga izobraževalna tradicija skoraj ne omenja. Ker pa je povsem odsoten tudi iz programa italijanščine kot tujega jezika na višji stopnji, je razumljivo, da se spričo "neobičajnih" jezikovnih kombinacij - nekatere zakonitosti rabe se v slovenščini in v italijanščini namreč bistveno razlikujejo - slovenski bralec ob branju italijanskega romana, za katerega je značilna izjemna pogostnost te retorične tehnike, upravičeno počuti nelagodno. Avtorica prispevka je raziskala principe Bassanijevega kombiniranja razpoložljivih parametrov in izhajajočo jezikovno podobo besedila ter opozarja na nekatere interpretacijske težave, na nekatere nedoslednosti $v$ pisateljevi rabi in utemeljuje nekatere kombinacijske posebnosti. 